\title{
A thermo-elastic-plastic phase-field model for simulating the evolution and transition of adiabatic shear band. Part II. Dynamic collapse of thick-walled cylinder
}

\author{
T. Wang ${ }^{\mathrm{a}}$, Z.L. Liu' ${ }^{\mathrm{a}, *}$, Y.N. Cui ${ }^{\mathrm{a}}$, X. Ye ${ }^{\mathrm{b}}$, X.M. Liu' ${ }^{\mathrm{b}}$, R. Tian ${ }^{\mathrm{c}}$, Z. Zhuang ${ }^{\mathrm{a}, *}$ \\ a Applied Mechanics Lab., School of Aerospace Engineering, Tsinghua University, Beijing 100084, China \\ ${ }^{\mathrm{b}}$ LNM, Institute of Mechanics, Chinese Academy of Sciences, Beijing 100190, China \\ ${ }^{\mathrm{c}}$ Software Center for High Performance Numerical Simulation, Institute of Applied Physics and Computational Mathematics, Beijing 100088, China
}

\section{A R T I C L E I N F O}

\section{Keywords:}

Adiabatic shear band

Phase-field

Thick-walled cylinder

Self-organizing behavior

Defect effect

\begin{abstract}
A B S T R A C T
In Part I, a thermo-elastic-plastic phase-field model is established for describing the adiabatic shear band (ASB) in metal materials. In this Part II, the developed model is used to simulate the classical thick-walled cylinder (TWC) experiment to investigate the self-organizing behavior of multiple ASBs. For the first time, the formation process of self-organized ASBs in the TWC experiment is reproduced by the phase-field method and the underlying physical mechanism is analyzed in detail. The simulation results show that the number and spacing of ASBs are related to loading rate and material properties. A higher loading rate leads to more intensive ASBs. For typical engineering materials such as 304L stainless steel (Ss304L) and titanium alloy (Ti6Al4V), the contribution of thermal softening to the formation of ASBs is far less than that of damage softening. However, thermal softening is very important to induce initial ASBs. In addition, we also find that defects, especially large ones, play a dominant role in the initiation and evolution of ASBs, leading to complex patterns of ASBs in the TWC experiments.
\end{abstract}

\section{Introduction}

Thermal-assisted shear localization is one of the most important deformations and failure mechanisms of materials under impact loading, which widely exists in high-speed impact, explosion, high-speed forming, erosion, penetration, and other high strain rate processes $[1,2]$. In the past studies, more attention has been paid to the single ASBs $[3,4]$. However, in dynamic deformation events, it is often found that multiple ASBs occur and interact at the same time. For example, in the typical TWC experiment, the character spacing and mode of the shear band have the characteristics of self-organization [5-7]. As shown in Fig. 1, these self organized ASBs have been observed in the TWC experiments for different materials with different specimen sizes, such as in Ss304L [7], Ti6Al4V [8], tantalum [9], 7075 aluminum alloy [2], and so on. The basic and quantitative understanding of the evolution law of the position, spacing, and width of these self-organized ASBs is of guiding significance for controlling material failure and utilizing ASBs.

Early researches and predictions of multiple ASBs were based on one-dimensional (1D) models, and different strength and failure models were proposed to predict the spacing between ASBs [10-12]. Grady and Kipp [13] first deduced the evolution spacing of ASBs by minimizing the energy and got the theoretical formula. Wright and Ockendon [11] and Molinari [12] used perturbation analysis method to analyze the spacing of ASBs. Zhou et al. [14,15] systematically studied the influence factors of the spacing of ASBs by $1 \mathrm{D}$

\footnotetext{
* Corresponding authors.

E-mail addresses: liuzhanli@tsinghua.edu.cn (Z.L. Liu), zhuangz@tsinghua.edu.cn (Z. Zhuang).
} 


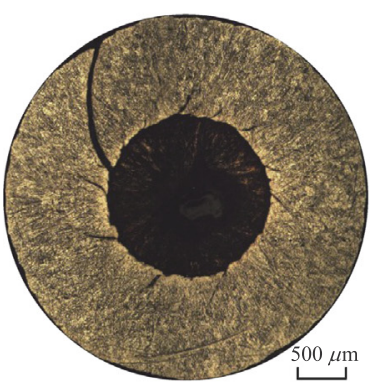

(a) Ss304L

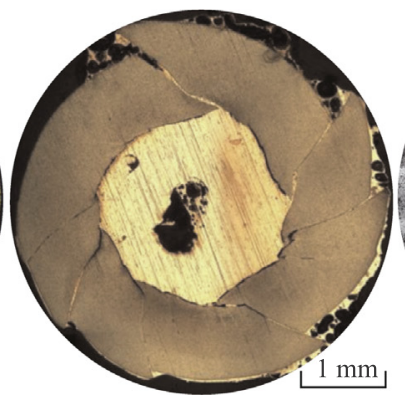

(b) Ti6Al4V

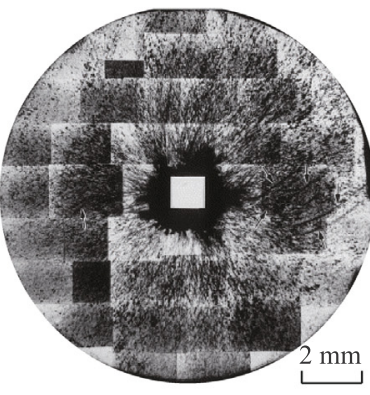

(c) Tantalum

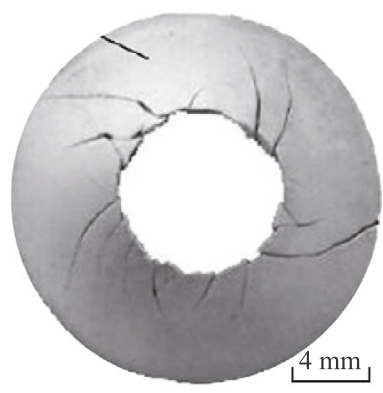

(d) 7075 aluminum alloy

Fig. 1. Distribution of self-organized ASBs in dynamic collapse TWC experiment of different materials: (a) Ss304L [7], (b) Ti6Al4V [8], (c) tantalum [9], and (d) 7075 aluminum alloy [2].

numerical analysis and gave empirical formula. They all gave simple formulas for calculating the spacing of ASBs, which provided a basis for the prediction of the TWC experiments and the verification of numerical models [16,7]. It is believed that these 1D models have the abilities to predict the spacing of ASBs in the experiments to some extent. However, these 1D models cannot reproduce the complex self-organizing behavior of ASBs in two-dimensional (2D) and three-dimensional (3D) conditions.

To study the ASBs more carefully and reproduce more realistic results of the TWC experiment, a large number of prediction studies on ASBs have been carried out by using 2D and 3D numerical models. Areias and Belytschko [17] proposed a two-scale model to simulate ASBs in the TWC experiment using extended finite element method (XFEM), and the simulation results were qualitatively consistent with the experiment. Rabczuk and Samaneigo [18] simulated 3D ASBs in the TWC experiments and the ASB was treated as a discontinuity and its width was ignored. These 2D and 3D numerical works $[19,17,18]$ explained the discontinuity of ASBs' spacing. However, they still cannot well describe the physical process of multi ASBs' formation because of lacking the physically based model for ASBs' evolution. In recent years, more complicated constitutive models have been adopted. The computational frameworks based on damage mechanics were proposed [20-22], which generally required a specific criterion to decide the initiation and propagation of the dynamics ASBs. These models are mainly based on the evolution of local damage variables, which can reflect the evolution of the entire ASB and provide us with in-depth insights into the self-organization behavior of ASBs. However, the mesh dependence and damage parameter determination of the damage based model to simulate the ASBs have not been well resolved.

The phase-field method is a very effective method to study the complex coupled fracture and failure problems [23-29], including shear failure of materials [30-32]. In Part I, a thermo-elastic-plastic phase-field model was established to simulate the ASBs. The damage parameters of the phase-field model were calibrated through experimental data, in contrast to the micro-physical mechanism-based damage model [33-37]. In this Part II, we use the developed phase-field model to study self-organized ASBs in the TWC experiment. To the best of our knowledge, the phase-field method has not been used to modeling the TWC experiment. The effects of double softening, loading rate, defect, mesh, and initial perturbation on the formation of self-organized ASBs are investigated.

The paper is organized as follows: the problem description and numerical model are presented in Section 2. The simulation results of the TWC experiment and verification are given in Section 3, including the comparisons with the theoretical formulas and experimental results. The physical mechanism of ASBs' evolution, such as damage softening, thermal softening and defect effect, is studied in Section 4. The concluding remarks are given in Section 5.

\section{Problem statement}

In part I, we developed a thermo-elastic-plastic phase-field model. In this section, we will briefly review the governing equations of the phase-field model and apply them to the numerical study of the TWC experiment.

\subsection{Governing equations}

The governing equations of the thermo-elastic-plastic phase-field model are as follows.

Momentum balance equation

$$
\operatorname{div} \sigma+\rho \boldsymbol{b}=\rho \ddot{\boldsymbol{u}} \quad \text { in } \Omega \times\left[0, t_{f}\right]
$$

Evolution equation of phase-field variable $d[38,32]$

$$
\eta \dot{d}=\left\{\begin{array}{lll}
\left\langle(1-d)\left\langle\psi_{e 0}^{+}+\psi_{p 0}-(1-\chi) w_{0}\right\rangle-\frac{g_{c s}}{2 l_{c}}\left(d-l_{c}^{2} \Delta d\right)\right\rangle_{+}, & d<1 & \text { in } \Omega \times\left[0, t_{f}\right] \\
0, & \text { otherwise }
\end{array}\right.
$$

Energy balance equation (i.e., evolution equation of temperature field) [39,40] 


$$
\rho c \dot{\theta}+\nabla \cdot \boldsymbol{J}=\gamma \quad \text { in } \Omega \times\left[0, t_{f}\right]
$$

The isotropic hardening $J_{2}$ flow model of von Mises yield surface is adopted. The Johnson-cook (JC) model is used to describe the yield of materials

$$
\sigma_{Y}^{0}=\left(A+B \varepsilon_{p}^{N}\right)\left(1+C \ln \frac{\dot{\varepsilon}_{p}}{\dot{\varepsilon}_{0}}\right)\left(1-\left(\frac{\theta-\theta_{0}}{\theta_{m}-\theta_{0}}\right)^{m}\right)
$$

The initial condition of the field variables:

$$
\boldsymbol{u}(\cdot, 0)=\boldsymbol{u}_{0}, \quad \dot{\boldsymbol{u}}(\cdot, 0)=\boldsymbol{v}_{0}, \quad \theta(\cdot, 0)=\theta_{0}, \quad d(\cdot, 0)=d_{0},
$$

where $\rho$ is the mass density, $\boldsymbol{b}$ is the volume force vector, $\Omega$ is the domain of solution, $t_{f}$ is the total solution time. $\chi \in(0,1]$ is the fraction of plastic work converted to thermal energy. It is usually considered as a constant and independent the material. In this paper, we assume that $\chi=0.9[30,39,41] . \eta$ is the viscosity parameter. $l_{c}$ is the characteristic width of the ASB. $g_{c s}$ is the material parameter that characterizes the evolution of phase-field. $w_{0}$ is a damage parameter, which determines the energy of damage initiation. $c$ is the specific heat, $\gamma$ is the body heat source per unit volume, and $\boldsymbol{J}$ is the internal heat flux which is assumed to be proportional to the temperature gradient: $\boldsymbol{J}=-k \cdot \nabla \theta$. Here $k$ is the thermal conductivity. $\sigma_{Y}^{0}$ is the yield stress of undamaged material, $A, B, C, N$ and $m$ are the material parameters in the JC model, $\theta_{m}$ is the melting temperature and $\theta_{0}$ is the reference temperature, 0.0 ${ }^{\circ} \mathrm{C}$ here. $\dot{\varepsilon}_{0}$ is a reference strain rate and is taken as $\dot{\varepsilon}_{0}=1.0 \mathrm{~s}^{-1} \cdot \boldsymbol{u}_{0}, \boldsymbol{v}_{0}, \theta_{0}$ and $d_{0}$ are the initial displacement, velocity, temperature and phase field, respectively.

\subsection{Numerical model}

Compression of thick-walled cylinders is a classical problem of adiabatic shear bands. In this section, we consider a thick-walled cylinder (304L stainless steel (Ss304L) or titanium alloy (Ti6Al4V)), with an outer diameter of $41.0 \mathrm{~mm}$ and an inner diameter of $23.0 \mathrm{~mm}$. The cylinder is sandwiched between two copper ( $\mathrm{Cu}$ ) cylinders (exterior driver tube and interior stopper tube), as shown in Fig. 2(a). The exterior copper cylinder's diameter is $45.0 \mathrm{~mm}$ and interior copper cylinder's diameter is $20.0 \mathrm{~mm}$, respectively. Initially, there is a gap of $0.1 \mathrm{~mm}$ between the three cylinders. Under loading, they can contact each other and also allow separation. In order to prevent the interior copper cylinder from being collapsed to severe distortion, we placed an analytical rigid surface with a diameter of 0.1 times the inner diameter of the interior copper cylinder in the middle of the model (i.e., $D_{\text {rigid }}=2.0 \mathrm{~mm}$ ). During the initiation and evolution of the ASBs, the symmetry of the system will be destroyed. Therefore, we use the full model instead of the $1 / 2$ or $1 / 4$ model.

The external boundary of exterior copper is subjected to the boundary pressure which decays exponentially with time, i.e.,

$$
p(t)=p_{0} \cdot \exp \left(-\frac{t}{t_{f}}\right)
$$

as shown in Fig. 2(b). The peak pressure $p_{0}$ is $1.0 \mathrm{GPa}$ and the duration $t_{f}$ is $50 \mu \mathrm{s}$. The impulse acting on the exterior boundary is

$$
I=\int_{0}^{t_{f}} \pi D_{o} t_{c} \cdot p(t) d t=\pi D_{o} t_{c} p_{0} t_{f}\left(1-e^{-1}\right)
$$

where $D_{o}$ is the diameter of the exterior boundary, $t_{c}$ is the thickness of the cylinder.

The material parameters of the Ss304L, Ti6Al4V, and Cu are listed in Table 1. Other parameters such as heat conduction parameters and damage parameters are shown in Table 2. Both structural and unstructured meshes are used. A detailed discussion of the simulation results and mesh sensitivity under different mesh types and densities can be found in Section 3.5. We find that numerical

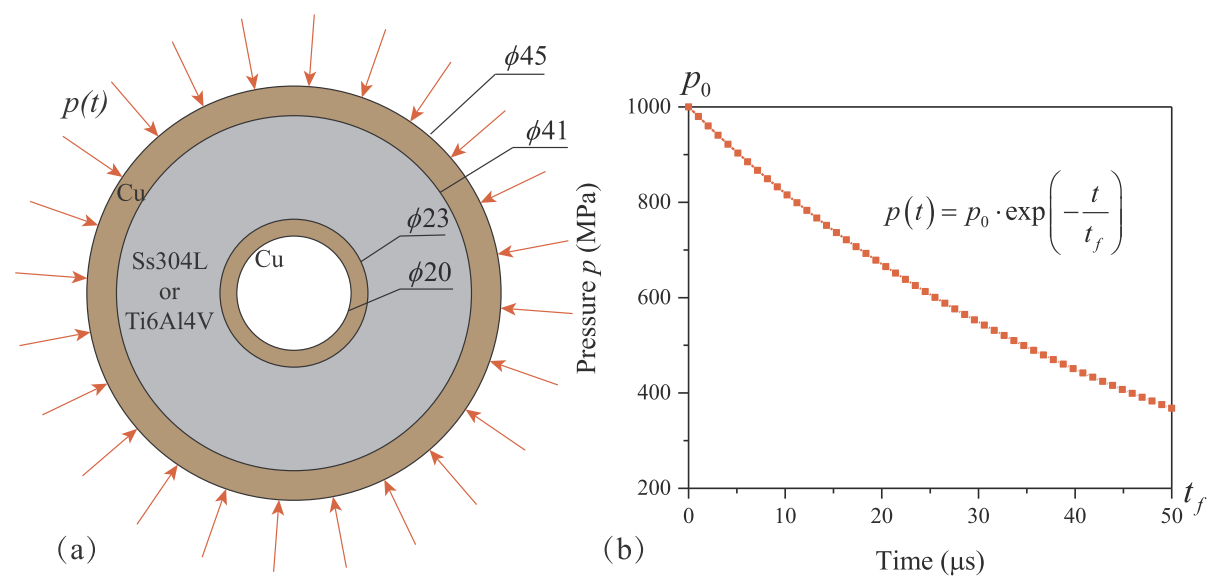

Fig. 2. The schematic diagram of the numerical model. (a) Configuration of the sandwich structure, and (b) curve of boundary pressure versus time. 
Table 1

The material parameters of JC model for metals [22,42,43].

\begin{tabular}{cccccccccc}
\hline & $\rho_{0}\left[\mathrm{~kg} / \mathrm{m}^{3}\right]$ & $E[\mathrm{GPa}]$ & $\nu$ & $A[\mathrm{GPa}]$ & $B[\mathrm{GPa}]$ & $C$ & $N$ & $m$ & $\theta_{\mathrm{m}}\left[{ }^{\circ} \mathrm{C}\right]$ \\
\hline Ss304L & 7900.0 & 210.0 & 0.29 & 0.1 & 1.072 & 0.05 & 0.34 & 1.0 \\
Ti6Al4V & 4430.0 & 110.0 & 0.33 & 0.862 & 0.331 & 0.012 & 0.34 & 0.8 & 1083.0 \\
Cu & 8920.0 & 120.0 & 0.2 & 0.09 & 0.292 & 0.025 & 0.31 & 1.09 & 1063.0 \\
\hline
\end{tabular}

Table 2

The other material parameters for metals $[22,42,43]$.

\begin{tabular}{cccccc}
\hline & $k\left[\mathrm{~W} / \mathrm{m}^{\circ} \mathrm{C}\right]$ & $c\left[\mathrm{~J} / \mathrm{kg}^{\circ} \mathrm{C}\right]$ & $\alpha_{\theta}\left[10^{-6} /{ }^{\circ} \mathrm{C}\right]$ & $g_{c s}[\mathrm{~N} / \mathrm{mm}]$ & $w_{0}\left[\mathrm{~J} / \mathrm{mm}^{3}\right]$ \\
\hline Ss304L & 16.2 & 500.0 & 17.3 & 2.0 & 0.15 \\
Ti6Al4V & 6.6 & 670.0 & 9.0 & 1.0 & 0.14 \\
$\mathrm{Cu}$ & 147.0 & 380.0 & 16.7 & 1.2 & 0.105 \\
\hline
\end{tabular}

perturbations are sufficient to induce asymmetric deformations of structures without the artificial introduction of other perturbations to break the symmetry. The effects of initial perturbations and defects on the evolution of ASBs will be discussed in detail later. The characteristic length scale parameter $l_{c}=0.1 \mathrm{~mm}$ and the mesh size $h=l_{c} / 2$. The viscous parameter $\eta$ is taken as $5.0 \times 10^{-8} \mathrm{kN} \cdot \mathrm{s} / \mathrm{mm}^{2}$.

\section{Numerical results and verifications}

\subsection{Evolution of ASBs}

The distributions of phase-field (i.e., shear bands) at different times for $t_{f}=50 \mu$ s (loading rate $1 / t_{f}=2 \times 10^{4} \mathrm{~s}^{-1}$ ) are shown in Fig. 3. Note that the loading rate here is a reflection of the overall loading speed, which is different from the local strain rate of the material point. As the stress wave generated by external loading transfers to the interior, the initial damage occurs in the inner cylinder. With the evolution of damage, the damage localization gradually appears and ASBs are formed. The final ASBs show spiral trajectories and a periodic distribution. There are 18 "main" ASBs (longer than $2 / 3$ of the maximum possible length) at the final stage, as shown in Fig. 3(c). The spacing between them at the inner cylinder was $4.01 \mathrm{~mm}$.

The detailed process is as follows: in the beginning, the stress evolves uniformly. With the accumulation of disturbances, small asymmetric waves are gradually generated (Fig. 3(a)). Then, the disturbance is rapidly amplified and a large number of small local deformation bands, namely adiabatic shear bands, are formed (Fig. 3(b)). As time goes on, there is competition between adjacent shear bands. Only some of them can form larger ASBs and eventually form nearly uniformly distributed ASBs. Until several or dozens of large ASBs extend to the exterior boundary, the structure completely collapses (Fig. 3(c)). The angle between the ASBs and the loading direction (radial direction) is $0^{\circ}$ when the ASB is initiated. When it extends to the exterior boundary of the specimen, the angle between them is about $45^{\circ}$ (Fig. 3(c)). The above is also called the self-organized behavior of ASBs. By observing the density of ASBs at different stages, it can be found that the number of ASBs in the initial stage is much larger than the number of large ASBs in the final stage. This is consistent with the previous experimental and numerical results [5,8,22].

Fig. 4 shows the temperature distribution of the whole field at several typical times for the loading duration $t_{f}=50 \mu$ s (loading rate $1 / t_{f}=2 \times 10^{4} \mathrm{~s}^{-1}$ ). It can be seen that the evolution of temperature is consistent with that of ASBs, and some high temperature self-organized band structures will be formed. In the early stage of the initiation and evolution of the ASBs, there will be some
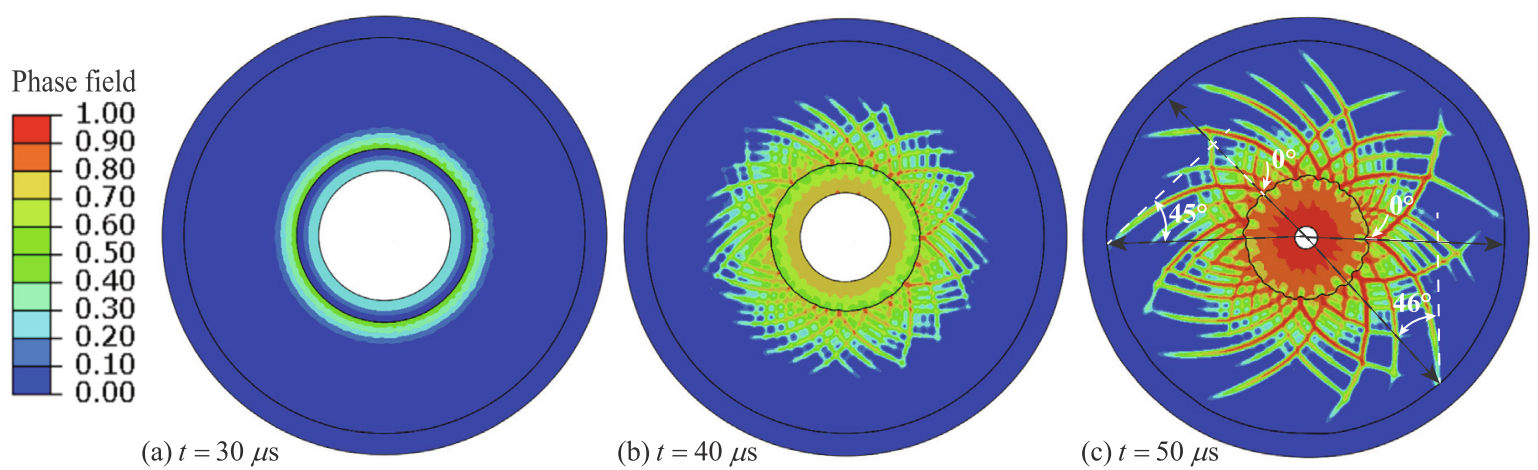

Fig. 3. The distribution of phase-field (i.e., shear bands) at different time for the loading duration $t_{f}=50 \mu$ s (loading rate $1 / t_{f}=2 \times 10^{4} \mathrm{~s}^{-1}$ ) with a time increment of $\Delta t=2.0 \times 10^{-4} \mu \mathrm{s}$. (a) $t=30 \mu \mathrm{s}$, (b) $t=40 \mu \mathrm{s}$, and (c) $t=50 \mu \mathrm{s}$. 

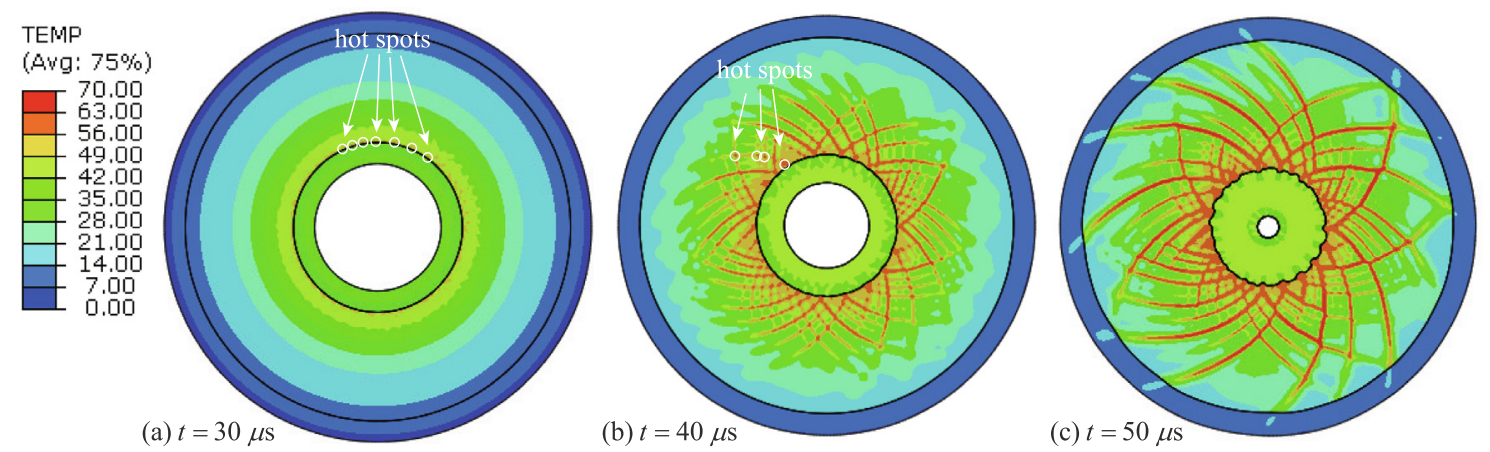

Fig. 4. The distribution of temperature field at different time for the loading duration $t_{f}=50 \mu \mathrm{s}$ with a time increment of $\Delta t=2.0 \times 10^{-4} \mu \mathrm{s}$. (a) $t=30 \mu \mathrm{s}$, (b) $t=40 \mu \mathrm{s}$, and (c) $t=50 \mu \mathrm{s}$. In the figure, discontinuous "hot spots" are formed in the early stage of the formation and evolution of the ASBs.

discontinuous "hot spots" (Fig. 4(a) and (b)), which are generally considered as the precursor of the rapid evolution of the ASBs. In other words, thermal softening plays an important role in the initiation of self-organized ASBs. This kind of discontinuous "hot spots" is also discussed in Part I. In addition, the final temperature distribution (Fig. 4(c)) is consistent with that of the final ASBs (Fig. 3(c)). That is, the temperature rise mainly occurs inside the ASBs, which is consistent with previous experimental observations [44] and simulation results $[39,41]$.

\subsection{Comparisons with theoretical solutions}

As we pointed out earlier, the theoretical formula of shear band spacing deduced by Grady and Kipp [13], Wright and Ockendon [11] and Molinari [12] can be used for prediction and numerical verification. The formulas they derived are as follows

$$
\begin{aligned}
& L_{G K}=2\left(\frac{9 k c}{\dot{\gamma}^{3} \alpha^{2} \tau_{0}}\right)^{1 / 4} \\
& L_{W O}=2 \pi\left(\frac{m^{3} k c}{\dot{\gamma}^{3} \alpha^{2} \tau_{0}}\right)^{1 / 4} \\
& L_{M}=2 \pi\left(\frac{m^{3} k c\left(1-\alpha \theta_{0}\right)^{2}}{\chi^{2}(1+m) \dot{\gamma}^{3} \alpha^{2} \tau_{0}}\right)^{1 / 4}
\end{aligned}
$$

where $\dot{\gamma}$ is the strain rate, $\tau_{0}$ is the flow stress, $\alpha$ is the thermal softening coefficient and $m$ is the linear strain-rate sensitivity coefficient.

In Fig. 5, the spacings of ASBs at different strain rates are simulated and compared with the theoretical solutions. It can be found that the numerical results are in good agreement with the theoretical formulas. In most cases, $L_{M}$ (or $L_{W O}$ ) $<L_{n u m}<L_{G K}$. In the final

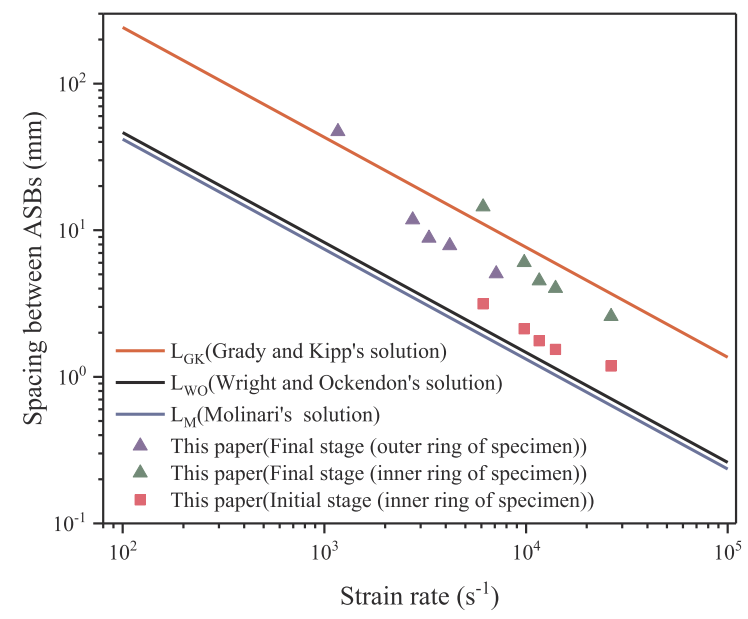

Fig. 5. The spacing between ASBs at different strain rates. Comparisons between simulation results and three theoretical solutions derived from Grady and Kipp [13], Wright and Ockendon [11] and Molinari [12]. 

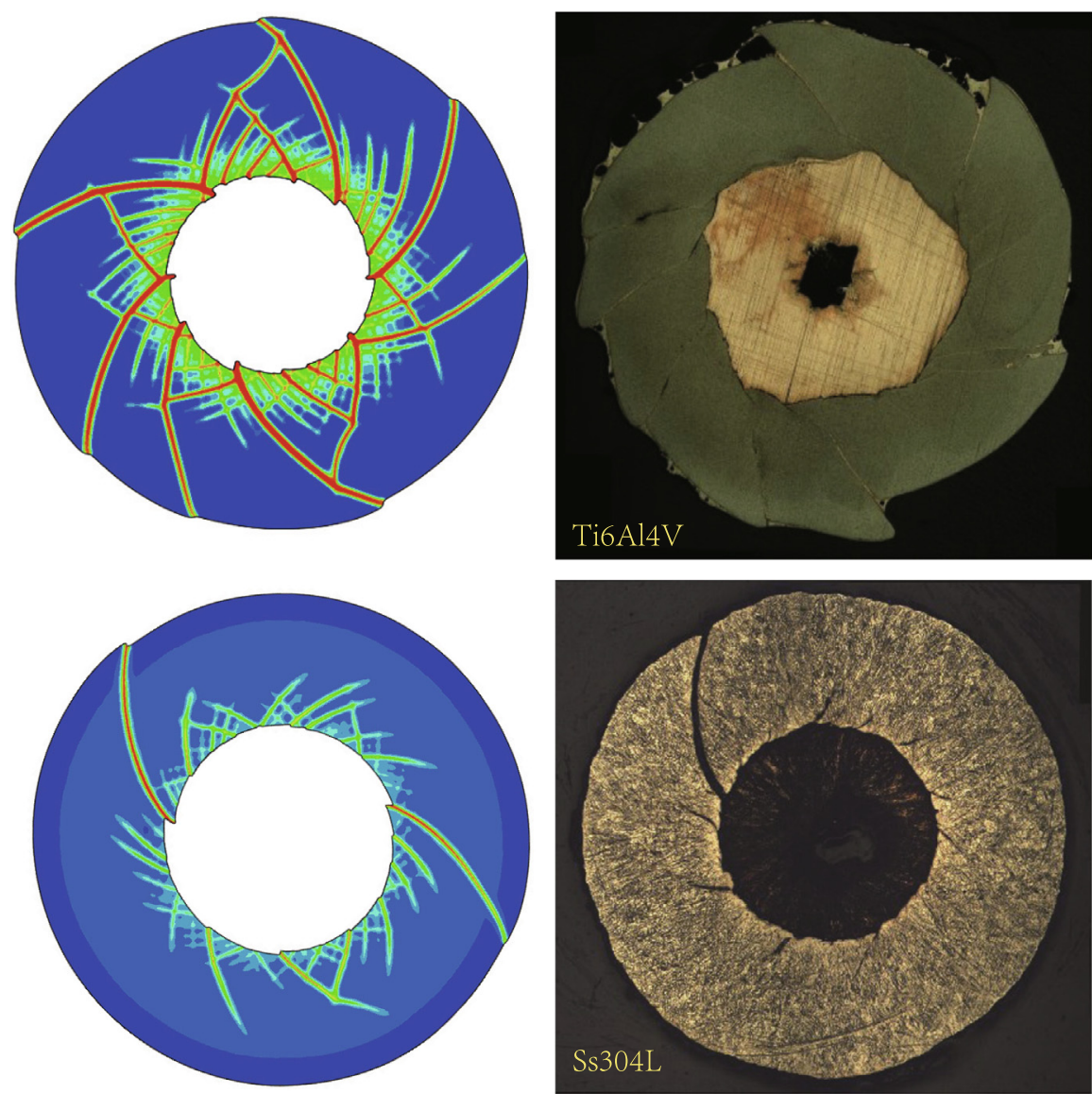

Fig. 6. Comparisons between simulation results (left) and two typical adiabatic shear bands in the experiment (right) with two different materials (Ss304L and Ti6Al4V) $[8,22]$.

stage of numerical simulation, the numerical solutions of the spacings between ASBs are closer to $L_{G K}$. Conversely, in the initial stage, the simulated ASBs' spacing is closer to $L_{W O}$ or $L_{M}$. This is because Wright and Ockendon's [11] and Molinari's [12] formulas based on perturbation analysis can be regarded as the lower bound of ASBs' spacing, which is usually more suitable for describing the initial stage of shear band initiation. While Grady and Kipp's [13] formula based on energy minimization is more suitable for describing the later stage. Previous experiments and numerical analysis also confirmed this point $[14,45]$.

It should be pointed out that the strain rate varies with the radius of the cylinder. However, at the same radius, the strain rate is the same at different moments. Therefore, the influence of strain rate on the spacing of shear bands can also be investigated through the distribution of shear bands at different radii of the same model.

\subsection{Comparisons with experimental results}

By adjusting the loading rate, we have successfully captured the distribution of ASBs for two different materials (Ss304L and Ti6Al4V) in the experiment, as shown in Fig. 6. The material parameters of the two materials are shown in Tables 1 and 2. For Ss304L, $t_{f}=70 \mu \mathrm{s}, p_{0}=0.714 \mathrm{GPa}$, and $\Delta t=2.0 \times 10^{-4} \mu \mathrm{s}$; for Ti6Al4V, $t_{f}=80 \mu \mathrm{s}, p_{0}=0.625 \mathrm{GPa}$ and $\Delta t=2.0 \times 10^{-4} \mu \mathrm{s}$. The simulation results of the final failure configuration and the number and direction of the ASBs agree well with the experimental results $[8,22]$.

Besides, the "jigsaw" displacement is observed in both numerical simulation and experiment, which is very typical in all experiments. One explanation for this displacement pattern is that the negative pressure of the shock wave causes the cylinder to move backwards. However, the boundary pressure in this paper has always been positive, which shows that the displacement pattern is mainly caused by the inconsistency of motion between the two sides of ASB due to shear localization. Moreover, the continuous description of discontinuities in the phase-field method makes the whole ASB in the calculated homogeneous material very smooth, which is consistent with the experimental observation. 
(a) Initial configuration

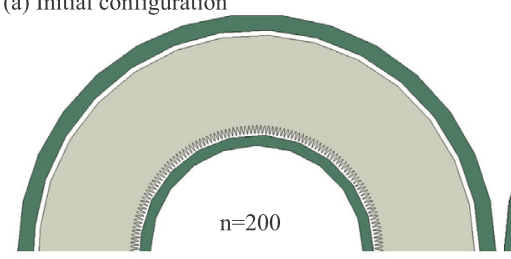

(b) Simulation results: ASBs' distribution

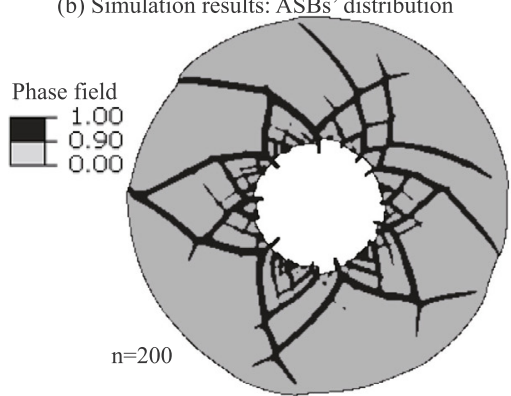

(c) Statistical distribution of ASBs' length

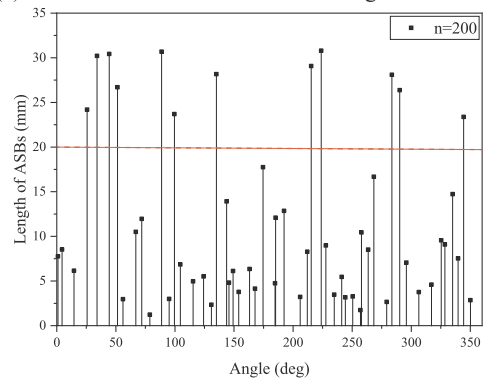

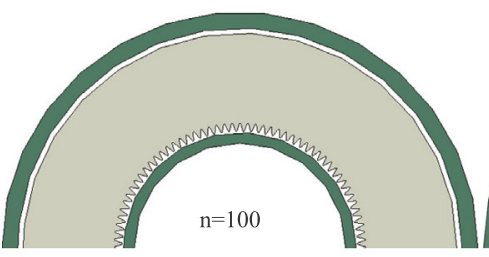
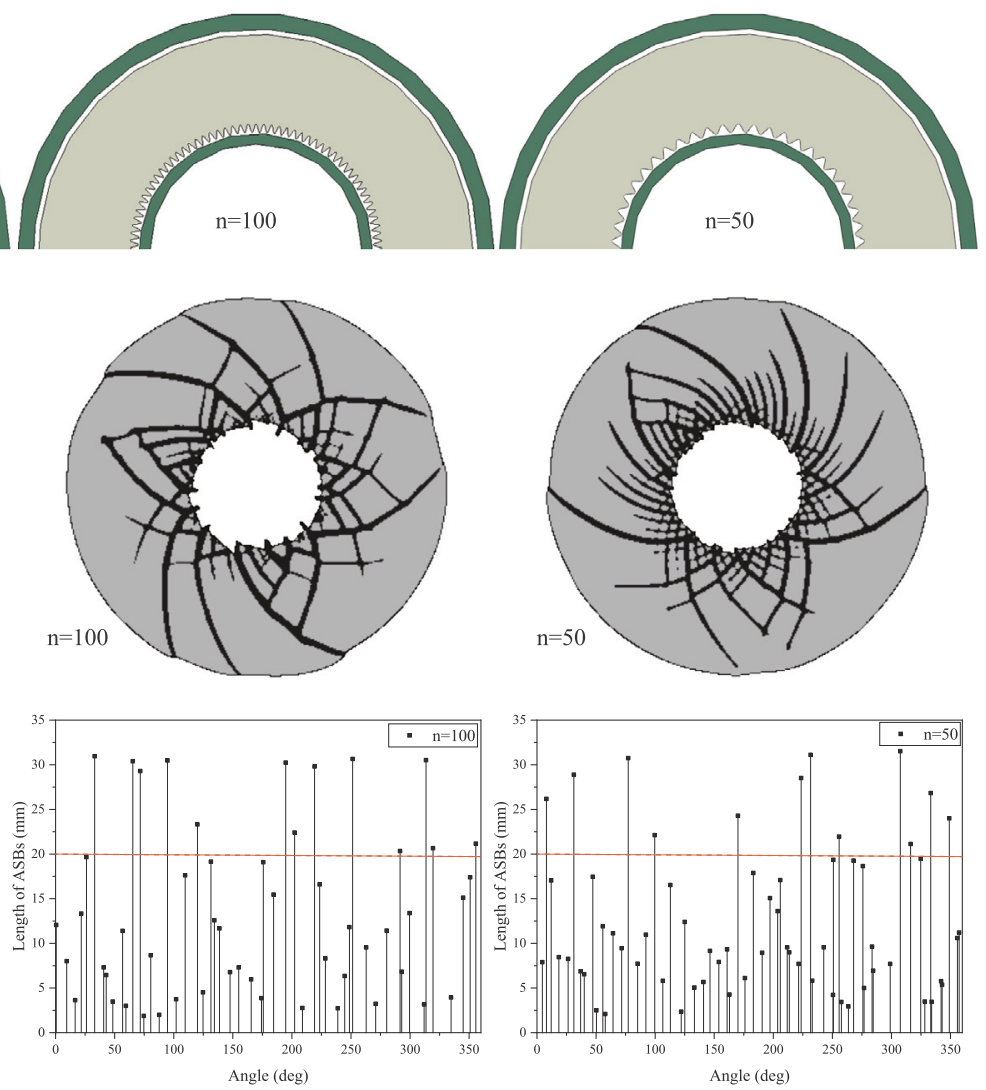

Fig. 7. The distribution of shear bands and their statistical values under different initial perturbations. (a) initial configuration (the perturbations are enlarged to show more clearly), (b) ASBs' distribution, and (c) the statistical distribution of the length of the ASBs.

\subsection{Initial perturbation analysis}

As we mentioned earlier, numerical perturbations break the symmetry and induce ASBs. We have previously studied the effects of very small perturbations (numerical perturbations) and very large perturbations (defects) on the evolution of ASBs. To further study the effects of the initial perturbation, we introduce a slight artificial perturbation in the model, which is stronger than the numerical perturbation, but much weaker than the defect, and has a certain regularity.

We give the initial perturbation of the following form:

$$
R_{\text {in }}(\theta)=R_{\text {in }}^{0}+A_{0} \cdot \cos (n \cdot \theta)
$$

where $R_{i n}^{0}$ and $R_{i n}(\theta)$ are the inner cylinder's radius of the sample before and after the initial perturbation, respectively. $n$ is the wave number on the perimeter. In the present study, we consider the case of $n=50,100$, and 200. $A_{0}$ is the amplitude of the initial perturbation. Its value is determined by the manufacturing tolerances of the sample and the microstructure and micro-defects inside the material. In the present study, we set $A_{0}=0.01 \mathrm{~mm} . \theta \in[0,2 \pi)$ is the angle of the point on the perimeter in polar coordinates. The initial configurations at different wave number $n$ are given in Fig. 7(a). It should be pointed out that we still use the whole model in the simulation, and the schematic diagram here gives half of it. The time increments of all the simulations in this section are set as $\Delta t=2.0 \times 10^{-4} \mu \mathrm{s}$.

The final distributions of the shear bands at different wave numbers $\mathrm{n}$ are shown in Fig. 7(b). With $d=0.9$ as the dividing line, the phase-field distribution is plotted as a black and white cloud for further statistical analysis. In all cases, the distribution of the shear bands looks very similar. For quantitative comparison, we sketched the shear bands in the simulation and plotted the length distribution of all shear bands along the perimeter, as shown in Fig. 7 (c). It can be seen that for $\mathrm{n}=200$, 100 and 50, the number of the large adiabatic shear bands (longer than $20 \mathrm{~mm}$ ) is 12, 13 and 12, respectively.

Further comparisons were made using the empirical cumulative distribution function (ECDF) [7,22]. ECDF does not follow the specific parameter form of the probability function, but instead generates a non-parametric density estimate to fit the raw data. The step function assigns only a probability of $1 / n$ to each of the observations in the specimen. The ECDF of the ASBs' length distribution under three wave numbers is shown in Fig. 8. Considering the probability characteristics of shear band formation, the comparison of ECDF shows that the three results at different wave numbers are actually similar. From these simulations, as long as the perturbation amplitude is small, the mode will not have a significant effect on the ASBs' distribution. On the other hand, for a sufficiently large 


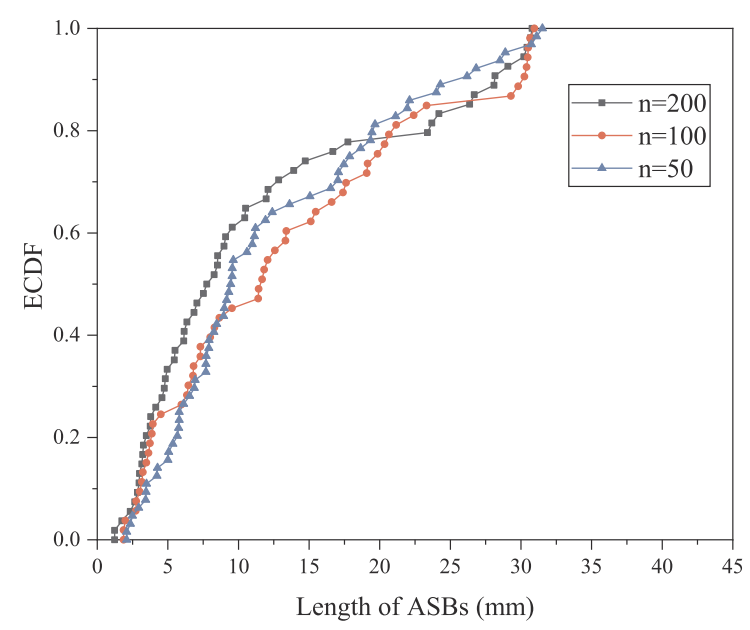

Fig. 8. The ECDF of ASBs' length for different wave number $n$ (corresponding to Fig. 7).

disturbance (such as a defect), the situation becomes similar to the case of a strongly fabricated core of the ASB, and the ASB dominates the region near the defect, as discussed in Section 4.3 in detail.

\subsection{Mesh sensitivity analysis}

Keeping other parameters unchanged (including $l_{c}=0.1 \mathrm{~mm}$ ), different mesh densities and mesh types were adopted to investigate the mesh sensitivity of the ASBs' distribution. Three kinds of mesh densities (represented by the feature size of the mesh) are used, and structural and unstructured meshes are adopted for each mesh density. Table 3 shows six different mesh densities, mesh types and corresponding number of elements, and the time increments used in the simulations.

The final distribution of shear bands for different mesh densities and mesh types is given in Fig. $9\left(t=t_{f}=60 \mu \mathrm{s}\right)$. It can be seen that under six conditions, the distribution of the final ASBs appears to be similar, but not exactly the same. Because different meshes will produce different numerical perturbations, it will affect the initiation of the shear band and thus affects the final distribution of the ASBs.

For quantitative comparison, we consider the statistical results of the number and length of ASBs to characterize the simulation results. Similarly, according to the statistical method mentioned in Section 3.4, the ECDF of the length distribution of the ASBs under different meshes is obtained (Fig. 10). The statistical results show that the distribution of ASBs under different mesh densities and mesh types is consistent. Table 4 lists the total number of shear bands and the number of large shear bands (length $L$ greater than 0.67 times the maximum length $L_{\max }$ ) calculated under six different conditions. It also shows that both the total number of ASBs and the number of large ASBs are consistent for different meshes. This further illustrates the consistency of the simulation results for different meshes.

The measurement of the shear band's width is very important and difficult in simulating the shear band problems. Shear band widths ranging from a few microns to a few hundred microns were observed in different TWC experiments. As the model proposed here introduced an intrinsic parameter $l_{c}$ characterizing the width of the shear band, the damage evolution in the width direction of the shear band could be simulated. The distribution of local shear bands for the results of the simulations under different meshes is given in Fig. 11. From the perspective of a local area of the model, the distribution of shear bands is random, and the distribution under different meshes is quite different. This does not contradict the mesh insensitivity of the entire ASBs' distribution discussed earlier. In fact, it is random in the local perspective but consistent in the overall perspective.

In Part I, we discussed the determination method of shear band width in detail based on the local deformation of the shear band. Here, we have also marked the width values of some typical shear bands in Fig. 11. The determination of the width of the shear band in the figure is determined by the degree of mesh distortion inside and outside the band. The mesh inside the shear band is severely distorted, creating a clearer boundary inside and outside the shear band. We found that the models under different meshes have different widths of shear bands at various locations. But most of their widths are around 200-300 $\mu$ m, about twice as much as $l_{c}$.

Table 3

Six different mesh densities, mesh types and corresponding number of elements, and the time increments used in the simulations.

\begin{tabular}{|c|c|c|c|c|c|c|}
\hline No. & $\# 1$ & $\# 2$ & $\# 3$ & $\# 4$ & \#5 & \#6 \\
\hline Mesh type & \multicolumn{3}{|c|}{ Structured mesh } & \multicolumn{3}{|c|}{ Unstructured mesh } \\
\hline Mesh size (mm) & 0.05 & 0.04 & 0.03 & 0.05 & 0.04 & 0.03 \\
\hline Number of elements & 498,644 & 777,988 & $1,387,090$ & 625,458 & 984,659 & $1,455,154$ \\
\hline Times increment $\Delta t(\mu \mathrm{s})$ & $2.0 \times 10^{-4}$ & $1.6 \times 10^{-4}$ & $1.2 \times 10^{-4}$ & $2.0 \times 10^{-4}$ & $1.6 \times 10^{-4}$ & $1.2 \times 10^{-4}$ \\
\hline
\end{tabular}



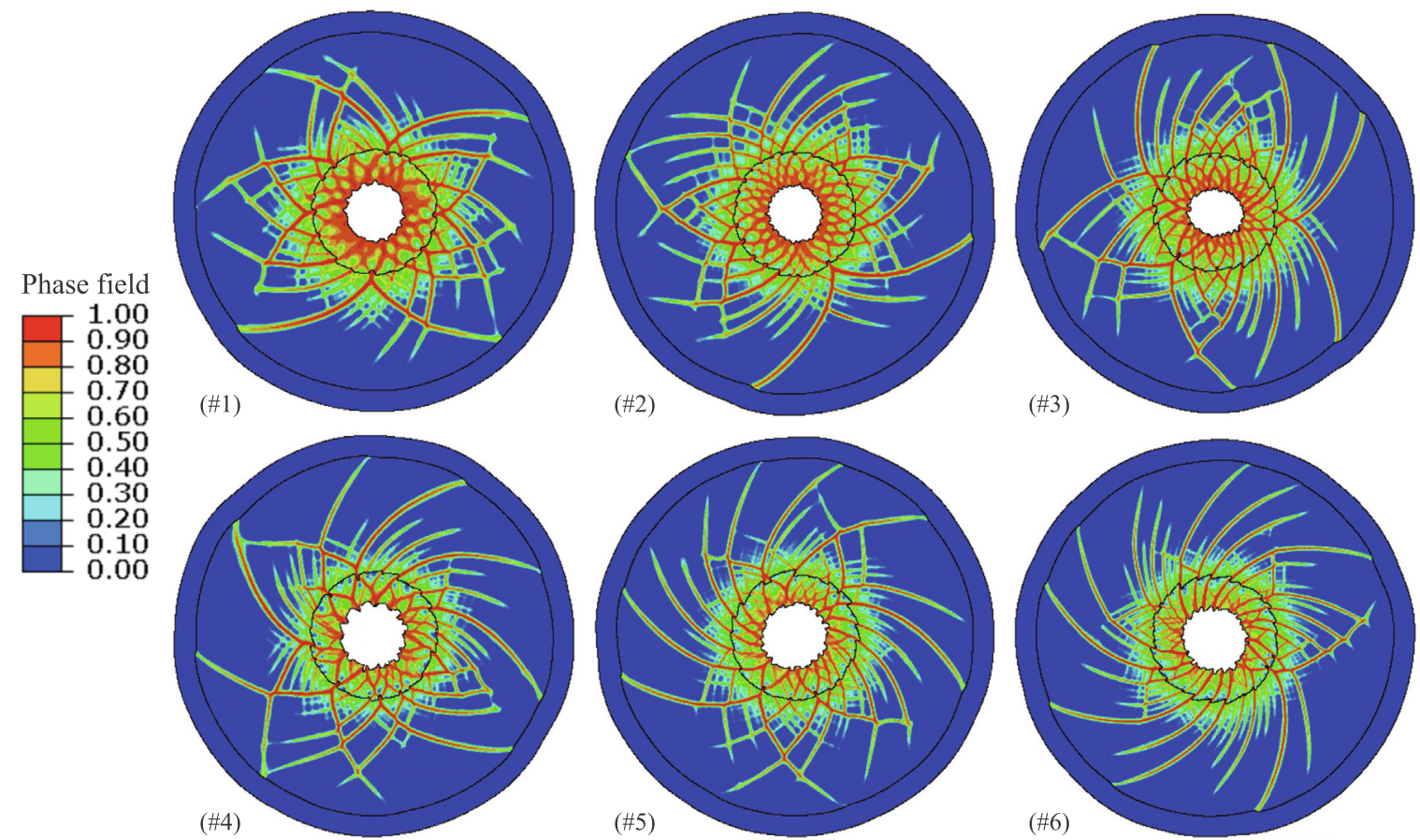

Fig. 9. The distribution of ASBs under different mesh types and densities. The corresponding mesh details are shown in Table 3.

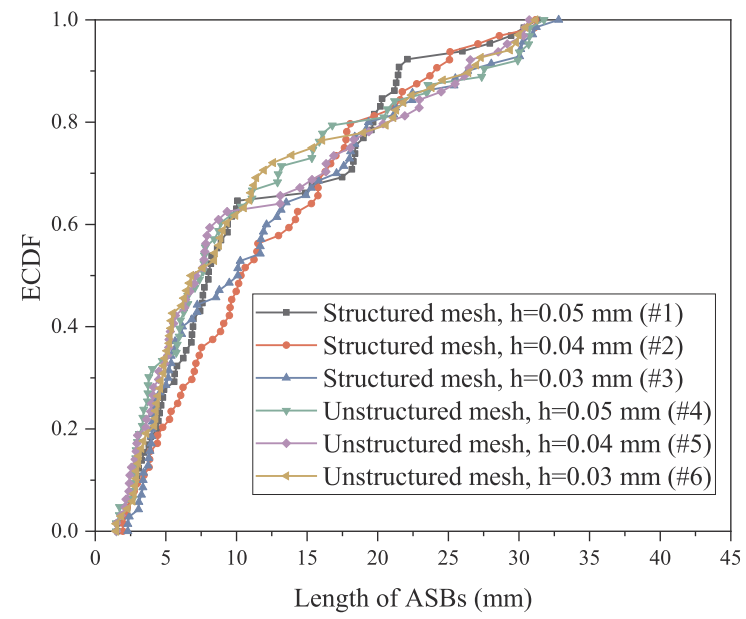

Fig. 10. The ECDF of ASBs' length for different mesh types and densities (corresponding to Fig. 9).

Table 4

Number of ASBs calculated under different meshes (statistical values).

\begin{tabular}{ccccccc}
\hline No. & $\# 1$ & $\# 2$ & $\# 3$ & $\# 4$ & \#5 & \\
\hline Total number of ASBs & 65 & 64 & 70 & 63 & 64 \\
Number of large ASBs $\left(L>\frac{2}{3} L_{\max }\right)$ & 12 & 11 & 13 & 12 & 13 & 14 \\
\hline
\end{tabular}

\section{Physical mechanism of ASBs' evolution}

\subsection{Role of thermal softening and damage softening}

The final ASBs, the corresponding Mises stress, and the temperature distribution of Ss304L for $t_{f}=50 \mu \mathrm{s}$ are shown in Fig. 12 (a) and (b). The increase of temperature mainly occurs at the shear band, which is why it is called an "adiabatic" shear band. In 

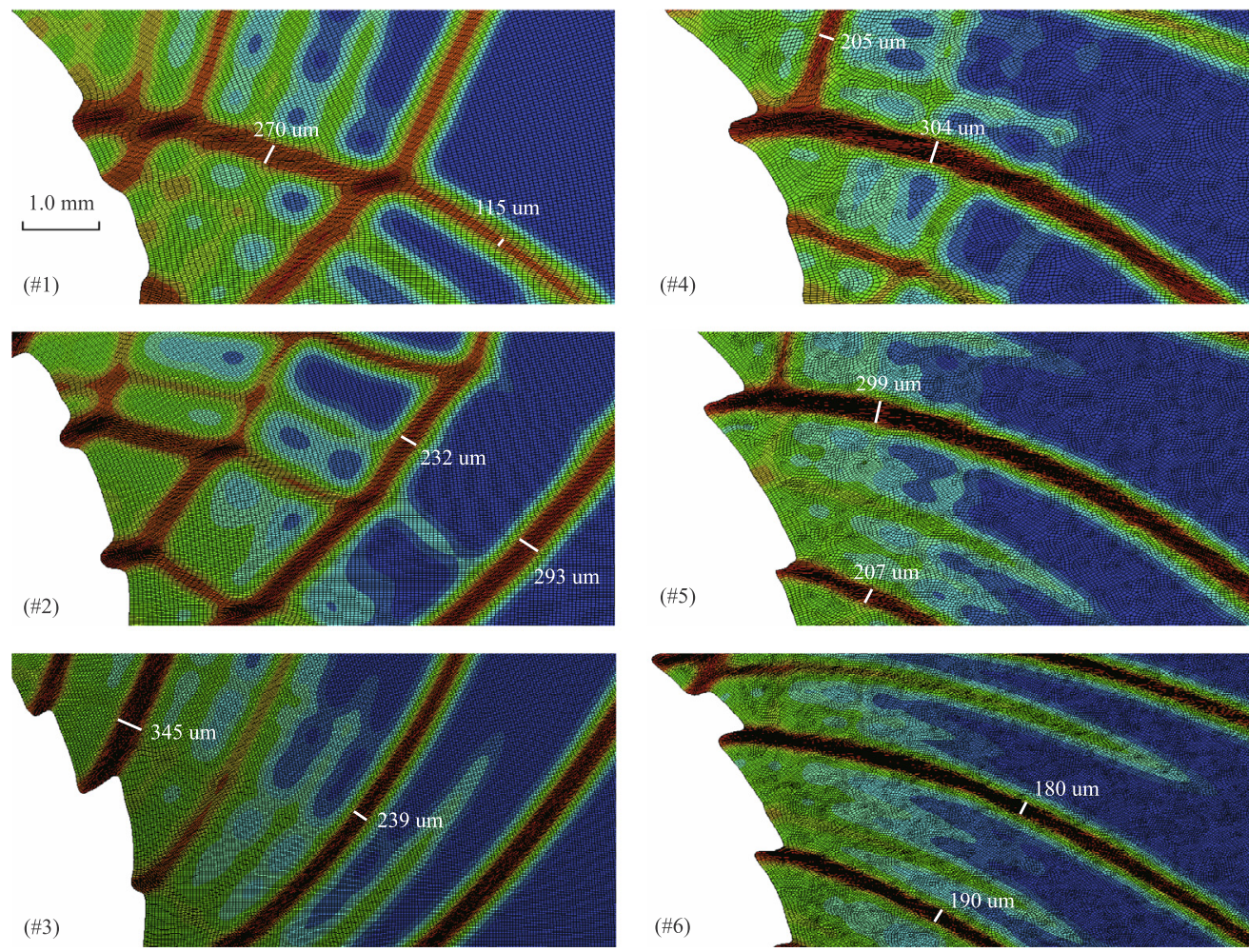

Fig. 11. Measurement of the width of the ASBs under different meshes.
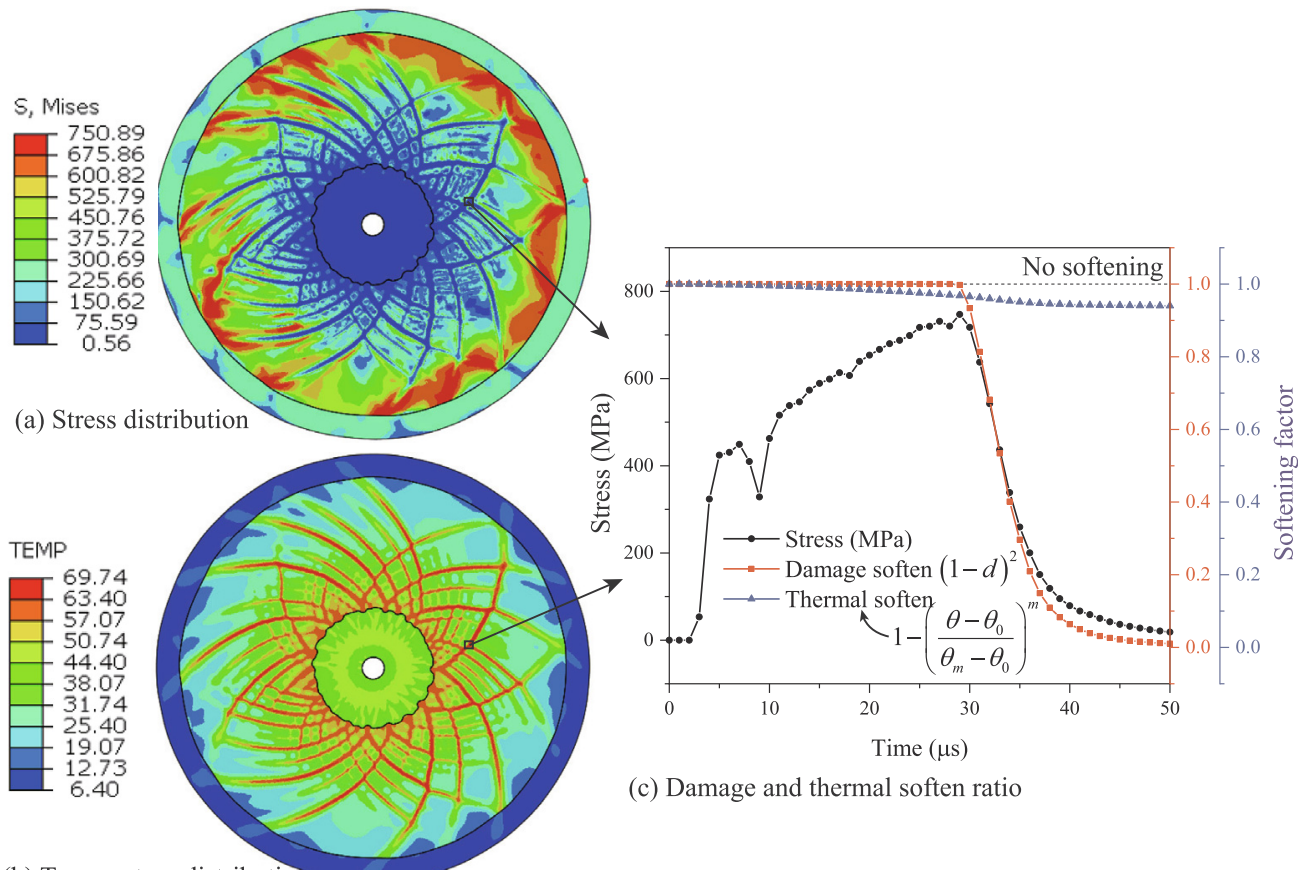

(c) Damage and thermal soften ratio

(b) Temperature distribution

Fig. 12. The final ASBs of Ss304L cylinder for $t_{f}=50 \mu \mathrm{s}$ with a time increment of $\Delta t=2.0 \times 10^{-4} \mu \mathrm{s}$. (a) Stress distribution (unit: MPa), (b) temperature distribution (unit: ${ }^{\circ} \mathrm{C}$ ), and (c) the Mises stress, thermal softening factor and damage softening factor of an element in the ASB versus time. 

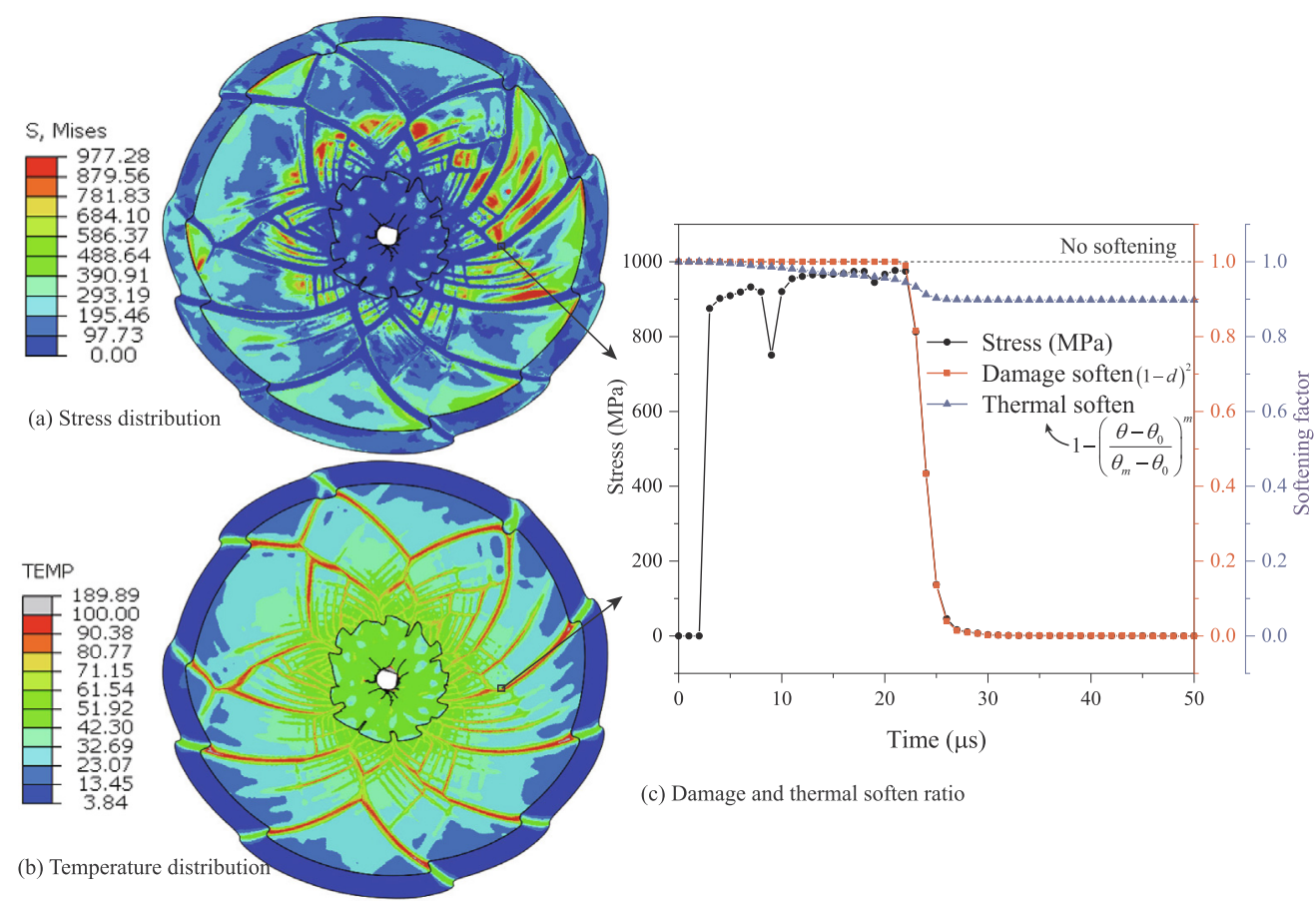

(c) Damage and thermal soften ratio

Fig. 13. The final ASBs of Ti6Al4V for $t_{f}=50 \mu$ s with a time increment of $\Delta t=2.0 \times 10^{-4} \mu \mathrm{s}$. (a) Stress distribution (unit: MPa), (b) temperature distribution (unit: ${ }^{\circ} \mathrm{C}$ ), and (c) the Mises stress, thermal softening factor and damage softening factor of an element in the ASB versus time.

particular, the rate of deformation localization and heat generation is very fast. In such a short period of time, the heat is too late to spread out, so the "adiabatic" effect is produced.

From the previous theoretical model, we know that the softening of materials is mainly caused by two mechanisms: thermal softening and damage softening. To quantitatively evaluate the effect of these two softening mechanisms, we present the variations of softening factor $\left((1-d)^{2}\right)$, thermal softening factor $\left(1-\left(\frac{\theta-\theta_{0}}{\theta_{m}-\theta_{0}}\right)^{m}\right)$, and the corresponding Mises stress of an element in the adiabatic shear band with time (Fig. 12(c)). Two phenomena are observed. (i) Thermal softening occurs prior to the damage softening. (ii) More importantly, damage softening contributes much more to the collapse of thick-walled cylinders and the evolution of ASBs than thermal softening. This indicates that thermal softening is not the dominant factor for the evolution of shear localization for the Ss304L cylinder.

Similar results were obtained for the material of Ti6Al4V. At $t_{f}=50 \mu \mathrm{s}$, the final ASBs, stress and temperature distribution, as well as the stress, damage softening, and thermal softening of Ti6Al4V as a function of time are shown in Fig. 13. Although the legend shows a maximum temperature rise of $189.89{ }^{\circ} \mathrm{C}$, the temperature rise in the main ASBs is about $90{ }^{\circ} \mathrm{C}$. For Ti6Al4V, damage softening also dominates the evolution of the shear band. The contribution of damage softening is much greater than the contribution of thermal softening (Fig. 13(c)).

Besides, some differences were observed in the evolution of ASBs between Ti6Al4V and Ss304L. Under the same loading conditions, the temperature rise in the ASB is higher. The contribution of thermal softening in Ti6Al4V is slightly higher than that of Ss304L. Once the damage of Ti6Al4V starts to evolve, it quickly evolves to 1.0, and the temperature rise mainly occurs within this short time. This is related to the material constitutive and damage evolution parameters $\left(w_{0}\right.$ and $\left.g_{c s}\right)$ of Ti6Al4V, as discussed in detail in Part I.

Although the proportion of thermal softening in the above two materials is small, it does not mean that thermal softening is not important in the initiation and evolution of the ASBs. In fact, for both materials, the thermal softening factor is greater than the damage softening factor in the early stages of ASBs initiation. In other words, thermal softening plays a dominant role in the early evolution of the ASBs. Due to thermal softening, a large number of initial ASBs are induced and eventually evolved into self-organized ASBs.

\subsection{Loading rate effect}

In the subsequent analysis, we study the effect of different loading rates on the formation of adiabatic shear bands by changing $t_{f}$ and keeping impulse constant. The final phase-field distributions (corresponding to ASBs) at different loading rates (corresponding to different impact duration $t_{f}$ ) are shown in Fig. 14. It can be found that the number and spacing of ASBs are sensitive to the loading rate. The faster the loading rate is, the higher the corresponding strain rate is, and the denser the ASBs are generated to release enough energy. It should be pointed out that the increase of the loading rate corresponds to the increase of peak pressure and strain 


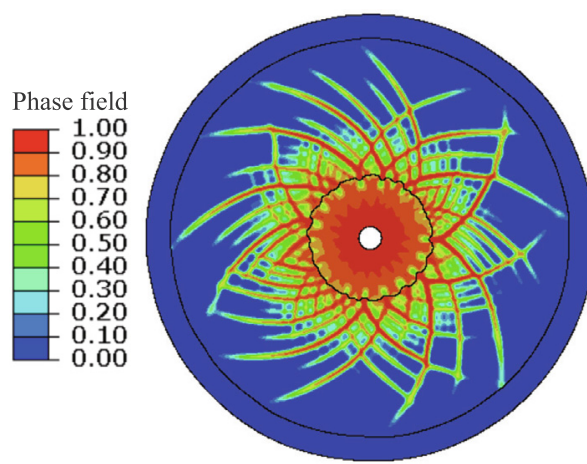

(a) loading rate $=2 \times 10^{4} \mathrm{~s}^{-1}$

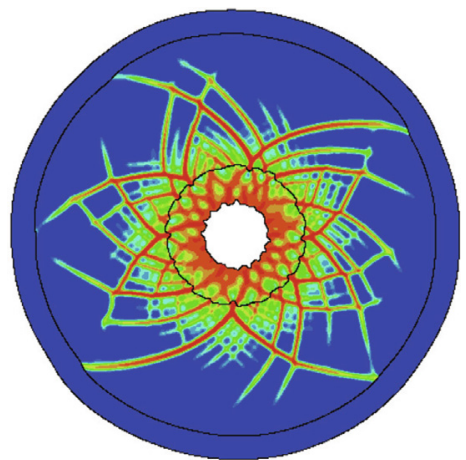

(b) loading rate $=1.67 \times 10^{4} \mathrm{~s}^{-1}$

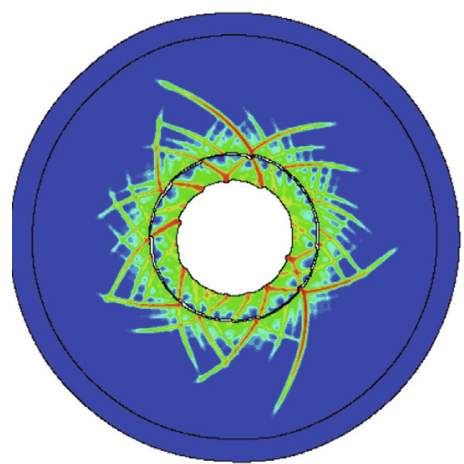

(c) loading rate $=1.43 \times 10^{4} \mathrm{~s}^{-1}$

Fig. 14. The distribution of the final ASBs under different loading rate $1 / t_{f}$. (a) $2.00 \times 10^{4} \mathrm{~s}^{-1}$, (b) $1.67 \times 10^{4} \mathrm{~s}^{-1}$ and (c) $1.43 \times 10^{4} \mathrm{~s}^{-1}$. The time increments of the simulations here are set as $\Delta t=2.0 \times 10^{-4} \mu \mathrm{s}$.

rate. Therefore, the influence of the loading rate can also be considered as the influence of peak pressure and strain rate.

At the same time, we also note that the number of adiabatic shear bands does not increase significantly when the loading rate is increased to a certain extent (Fig. 14a) and and Fig. 14(b), where the loading rate $1 / t_{f}=2 \times 10^{4} \mathrm{~s}^{-1}$ and $1.67 \times 10^{4} \mathrm{~s}^{-1}$ ). It is consistent with Grady's theory on the competition between kinetic energy and strain energy [46].

For different loading rates, we calculated the distribution of ECDF (Fig. 15). When the loading rate is high, the number of ASBs is more (the number of points on each line in Fig. 15), and the spacing of the ASBs is smaller. In addition, at a higher loading rate $\left(1 / t_{f} \geqslant 1.67 \times 10^{4} \mathrm{~s}^{-1}\right)$, the ASBs' distribution tends to be consistent with the length, that is, the proportion of ASBs in each length interval is consistent. On the contrary, at a lower loading rate $\left(1 / t_{f}=1.43 \times 10^{4} \mathrm{~s}^{-1}\right)$, the lengths of the ASBs are mainly distributed in a shorter length interval. That is, most of the ASBs are shorter under this condition.

\subsection{Defect effect}

Defects play an essential role in the initiation and evolution of ASBs, especially large ones. Adiabatic shear bands are often induced from defects. Therefore, we studied the effect of different defects on the formation of ASBs. Here, the formation of ASBs with 1, 2, 4 and 8 large defects is simulated. The location of the initial defects in each case is shown in Fig. 16(a). The size of each defect is $50 \mu \mathrm{m} \times 50 \mu \mathrm{m}$, which is achieved by deleting an element in the model. The time increments of all the simulations in this section are set as $\Delta t=2.0 \times 10^{-4} \mu \mathrm{s}$. The other model parameters are the same as the previous ones.

The simulation results for different number of defects are shown in Fig. 16(b-e). The region of $d \geqslant 0.95$ is removed to visualize the dominant ASBs. The results show that defects can induce larger ASBs. The area near the shear band will be shielded, forming a shielding zone without the shear bands. The area far from the defect is not affected. That is, in the case of large defects, defects will dominate the initiation and evolution of ASBs, and the main shear bands are extended from the defects. When the number of large defects is small, for example, 1 or 2 (as shown in Fig. 16(b) and (c)), there are still more ASBs with free initiation and propagation in the region far from the defects. When the number of defects is large, such as 4 or 8 (as shown in Fig. 16(d) and (e), defects should also

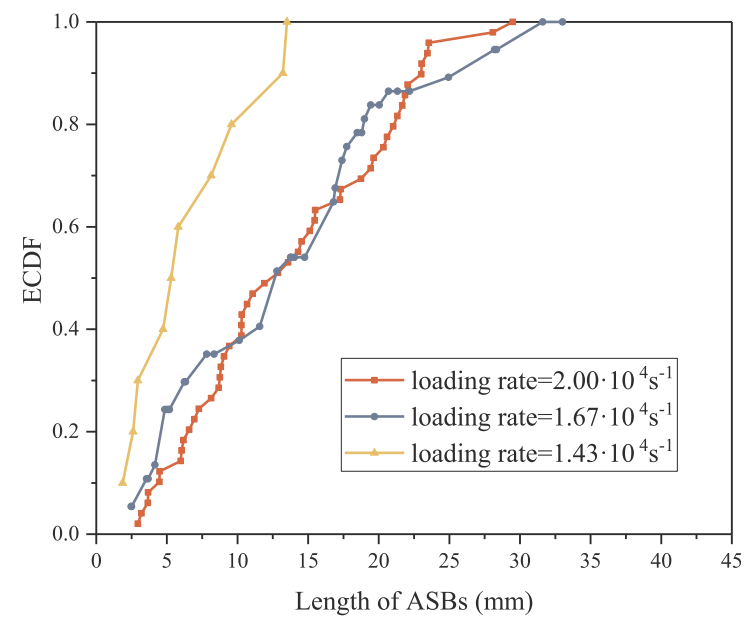

Fig. 15. The ECDF of ASBs length for different loading rate $1 / t_{f}\left(2.00 \times 10^{4} \mathrm{~s}^{-1}, 1.67 \times 10^{4} \mathrm{~s}^{-1}\right.$, and $1.43 \times 10^{4} \mathrm{~s}^{-1}$, corresponding to Fig. 14). The number of points on each line in the figure is the corresponding. number of ASBs. 
(a) Defect position for each case

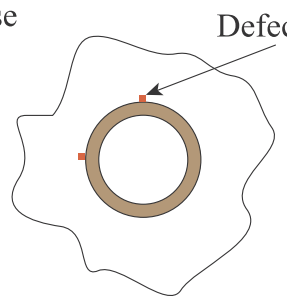

Defect position

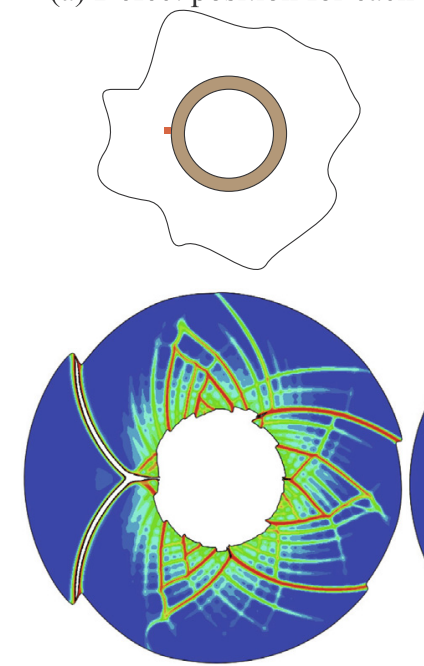

(b) One defect

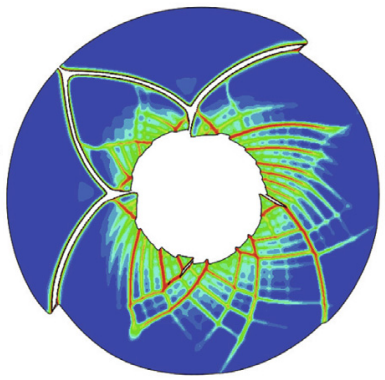

(c) Two defects

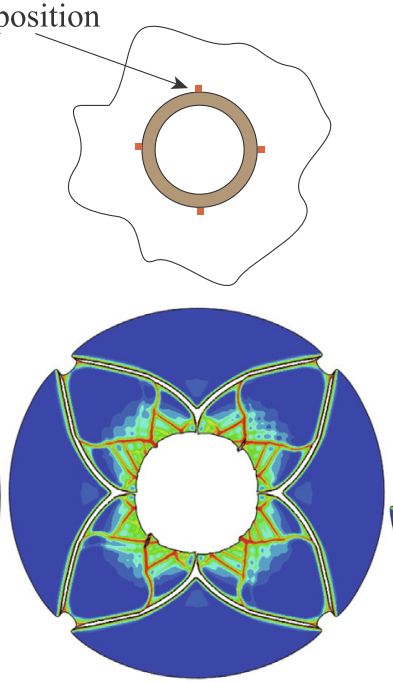

(d) Four defects
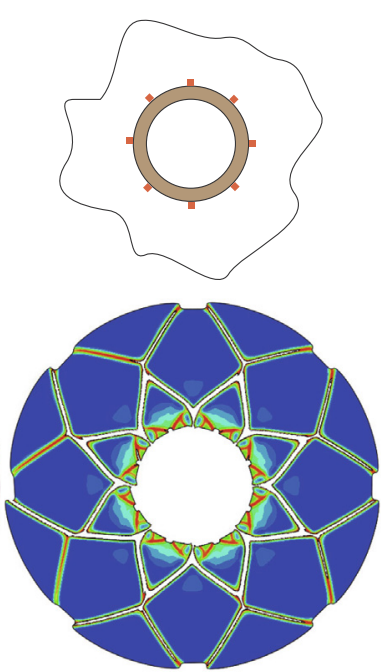

(e) Eight defects

Fig. 16. Distribution of ASBs simulated under different initial imperfections when $t_{f}=60 \mu$ s. (a) the defect position for each case, (b) simulated results for one defect, (c) simulated results for two defects, (d) simulated results for four defects, and (e) simulated results for eight defects. The region of $d \geqslant 0.95$ is removed to visualize the dominant ASBs.

be evenly distributed to shield larger areas), a more complex pattern of ASBs will be formed. The larger the number of large defects, the smaller the number of free-germinating ASBs.

The results here are useful in practice. We can fabricate defects of appropriate location and size to induce the desired ASBs. For example, in the aspect of penetration, the induced ASBs are very beneficial to the self-sharpening of penetrators.

\section{Conclusions}

In this paper, the complex dynamics of spontaneous adiabatic shear band evolution during the collapse of thick-walled cylinders is studied by a thermo-elastic-plastic phase-field model. The self-organizing behavior of ASBs has been investigated in detail. The distribution and spacing of ASBs are well described and quantitatively agreed with the theoretical formulas and experimental results.

It is found that the number and spacing of the ASBs are strongly correlated with the loading rate and material properties. A high loading rate will result in more ASBs and smaller spacing among ASBs. The results also show that damage softening contributes much more to the collapse of thick-walled cylinders and the propagation of ASBs than thermal softening for the given materials (Ss304L and Ti6Al4V). But thermal softening is very important in inducing the initial ASBs.

In addition, defects, especially large ones, play a dominant role in the initiation and evolution of ASBs, leading to complex patterns of ASBs. Initial perturbation analysis and mesh sensitivity analysis show that small perturbations, mesh densities, and mesh types do not affect the overall distribution of ASBs.

\section{Declaration of Competing Interest}

The authors declare that they have no known competing financial interests or personal relationships that could have appeared to influence the work reported in this paper.

\section{Acknowledgment}

This work was supported by Science Challenge Project (No. TZ2018002), the National Natural Science Foundation of China (Grant No. 11532008 and 11972208) and China Postdoctoral Science Foundation (No. 2019M650699).

\section{References}

[1] Rittel D. A hybrid experimental-numerical investigation of dynamic shear fracture. Eng Fract Mech 2005;72(1):73-89.

[2] Yang Y, Zeng Y, Gao ZW. Numerical and experimental studies of self-organization of shear bands in 7075 aluminium alloy. Mat Sci Eng A-Struct 2008;496(1):291-302.

[3] Li GA, Zhen L, Lin C, Gao RS, Tan X, Xu CY. Deformation localization and recrystallization in TC4 alloy under impact condition. Mat Sci Eng A-Struct 2005;395(1):98-101.

[4] Teng X, Wierzbicki T, Couque H. On the transition from adiabatic shear banding to fracture. Mech Mater 2007;39(2):107-25.

[5] Nesterenko VF, Meyers MA, Wright TW. Self-organization in the initiation of adiabatic shear bands. Acta Mater 1998;46(1):327-40. 
[6] Xue Q, Meyers MA, Nesterenko VF. Self organization of shear bands in stainless steel. Mat Sci Eng A-Struct 2004;384(1):35-46.

[7] Lovinger Z, Rikanati A, Rosenberg Z, Rittel D. Electro-magnetic collapse of thick-walled cylinders to investigate spontaneous shear localization. Int J Impact Eng 2011;38(11):918-29.

[8] Lovinger Z, Rittel D, Rosenberg Z. An experimental study on spontaneous adiabatic shear band formation in electro-magnetically collapsing cylinders. J Mech Phys Solids 2015;79:134-56.

[9] Chen YJ, Meyers MA, Nesterenko VF. Spontaneous and forced shear localization in high-strain-rate deformation of tantalum. Mat Sci Eng A-Struct 1999;268(1):70-82.

[10] Bai YL. Thermo-plastic instability in simple shear. J Mech Phys Solids 1982;30(4):195-207.

[11] Wright TW, Ockendon H. A scaling law for the effect of inertia on the formation of adiabatic shear bands. Int J Plasticity 1996;12(7):927-34.

[12] Molinari A. Collective behavior and spacing of adiabatic shear bands. J Mech Phys Solids 1997;45(9):1551-75.

[13] Grady DE, Kipp ME. The growth of unstable thermoplastic shear with application to steady-wave shock compression in solids. J Mech Phys Solids 1987;35(1):95-119.

[14] Zhou F, Wright TW, Ramesh KT. The formation of multiple adiabatic shear bands. J Mech Phys Solids 2006;54(7):1376-400.

[15] Zhou F, Wright TW, Ramesh KT. A numerical methodology for investigating the formation of adiabatic shear bands. J Mech Phys Solids 2006;54(5):904-26.

[16] Xue Q, Nesterenko VF, Meyers MA. Evaluation of the collapsing thick-walled cylinder technique for shear-band spacing. Int J Impact Eng 2003;28(3):257-80.

[17] Areias PMA, Belytschko T. Two-scale method for shear bands: thermal effects and variable bandwidth. Int J Numer Meth Eng 2007;72(6):658-96.

[18] Rabczuk T, Samaniego E. Discontinuous modelling of shear bands using adaptive meshfree methods. Comput Meth Appl Mech Eng 2008;197(6):641-58.

[19] Firstenberg O, Ashuach Y, Partom Y. A simple model for dynamic shear failure of stainless steel. J. Phys. IV France 2006;134:191-6.

[20] Daridon L, Oussouaddi O, Ahzi S. Influence of the material constitutive models on the adiabatic shear band spacing: MTS, power law and johnson-cook models. Int J Solids Struct 2004;41(11):3109-24.

[21] Dorothy HL, Longère P. Modelling of high strain rate adiabatic shear banding induced failure: a comparison of two approaches. Int J Impact Eng 2017;110:219-27.

[22] Lovinger Z, Rittel D, Rosenberg Z. Modeling spontaneous adiabatic shear band formation in electro-magnetically collapsing thick-walled cylinders. Mech Mater 2018;116:130-45.

[23] Nguyen T-T, Waldmann D, Bui TQ. Role of interfacial transition zone in phase field modeling of fracture in layered heterogeneous structures. J Comput Phys 2019;386:585-610.

[24] Zhang G, Guo TF, Zhou Z, Tang S, Guo X. A phase-field model for fracture in water-containing soft solids. Eng Fract Mech 2019;212:180-96.

[25] Geelen RJM, Liu Y, Dolbow JE, Rodríguez-Ferran A. An optimization-based phase-field method for continuous-discontinuous crack propagation. Int J Numer Meth Eng 2018;116(1):1-20.

[26] Geelen RJM, Liu Y, Hu T, Tupek MR, Dolbow JE. A phase-field formulation for dynamic cohesive fracture. Comput Meth Appl Mech Eng 2019;348:680-711. arXiv: 1809.09691.

[27] Wu J-Y, Mandal TK, Nguyen VP. A phase-field regularized cohesive zone model for hydrogen assisted cracking. Comput Meth Appl Mech Eng 2020;358:112614.

[28] Wu J-Y, Huang Y, Nguyen VP. On the BFGS monolithic algorithm for the unified phase field damage theory. Comput Meth Appl Mech Eng 2020;360:112704.

[29] Wang T, Ye X, Liu Z, Liu X, Chu D, Zhuang Z. A phase-field model of thermo-elastic coupled brittle fracture with explicit time integration. Comput Mech 2020:1-17.

[30] McAuliffe C, Waisman H. A unified model for metal failure capturing shear banding and fracture. Int J Plasticity 2015;65:131-51.

[31] Arriaga M, Waisman H. Combined stability analysis of phase-field dynamic fracture and shear band localization. Int J Plasticity 2017;96:81-119.

[32] Wang T, Ye X, Liu Z, Chu D, Zhuang Z. Modeling the dynamic and quasi-static compression-shear failure of brittle materials by explicit phase field method. Comput Mech 2019;64(6):1537-56.

[33] Feng XQ, Yu SW. Micromechanical modelling of tensile response of elastic-brittle materials. Int J Solids Struct 1995;32(22):3359-72.

[34] Feng XQ, Gross D. An approximate scheme for considering effects of microcrack interaction on the overall constitutive relation of brittle solids under complex loading. Acta Mech 2000;139(1):143-59.

[35] Feng XQ, Qin QH, Yu SW. Quasi-micromechanical damage model for brittle solids with interacting microcracks. Mech Mater 2004;36(3):261-73.

[36] Zeng Q, Tonge AL, Ramesh KT. A multi-mechanism constitutive model for the dynamic failure of quasi-brittle materials. Part I: Amorphization as a failure mode. J Mech Phys Solids 2019;130:370-92.

[37] Zeng Q, Tonge AL, Ramesh KT. A multi-mechanism constitutive model for the dynamic failure of quasi-brittle materials, Part II: Integrative model. J Mech Phys Solids 2019;131:20-42.

[38] Ziaei-Rad V, Shen Y. Massive parallelization of the phase field formulation for crack propagation with time adaptivity. Comput Meth Appl Mech Eng 2016;312:224-53.

[39] Chu D, Li X, Liu Z, Cheng J, Wang T, Li Z, Zhuang Z. A unified phase field damage model for modeling the brittle-ductile dynamic failure mode transition in metals. Eng Fract Mech 2019;212:197-209.

[40] Nguyen T-T, Waldmann D, Bui TQ. Computational chemo-thermo-mechanical coupling phase-field model for complex fracture induced by early-age shrinkage and hydration heat in cement-based materials. Comput Meth Appl Mech Eng 2019;348:1-28.

[41] McAuliffe C, Waisman H. A coupled phase field shear band model for ductile-brittle transition in notched plate impacts. Comput Meth Appl Mech Eng 2016;305:173-95.

[42] Zhang Y, Outeiro JC, Mabrouki T. On the selection of johnson-cook constitutive model parameters for ti-6al-4v using three types of numerical models of orthogonal cutting. Procedia CIRP 2015;31:112-7.

[43] Chen X, Du C. A gradient plasticity model for the simulation of shear localization. Adv Mech Eng 2017;9(9). 1687814017719005.

[44] Guo Y, Ruan Q, Zhu S, Wei Q, Chen H, Lu J, et al. Temperature rise associated with adiabatic shear band: causality clarified. Phys Rev Lett 2019;122(1):015503.

[45] Xue Q, Meyers MA, Nesterenko VF. Self-organization of shear bands in titanium and ti-6al-4v alloy. Acta Mater 2002;50(3):575-96.

[46] Grady DE. Adiabatic shear failure in brittle solids. Int J Impact Eng 2011;38(7):661-7. 